\title{
Linking global warming, metabolic rate of hematophagous vectors, and the transmission of infectious diseases
}

\section{Carmen Rolandi and Pablo E. Schilman*}

Departamento de Biodiversidad y Biología Experimental, Facultad de Ciencias Exactas y Naturales, Universidad de Buenos Aires, Buenos Aires, Argentina

*Correspondence: schilman@bg.fcen.uba.ar

Climate is under constant change. In addition to its natural variability, there is plenty of evidence suggesting persistent changes overtime produced by external forces, such as anthropogenic activities. These changes are observed on patterns of precipitation and temperature, among others (Crowley, 2000). Carbon dioxide levels in the atmosphere have been recorded since the beginning of the twentieth century. The hypothesis that changes in $\mathrm{CO}_{2}$ concentration of the atmosphere could be responsible for climate deviations, first proposed by Arrhenius (1896), is now known as the greenhouse effect. Much research has been done finding a connection between the rise of anthropogenic carbon dioxide (and other greenhouse-effect gases) to increases in the global mean temperatures. Energy balance model studies show that temperature changes during the past 100 years cannot be explained by natural factors alone (such as solar irradiation and volcanism). Global mean temperatures have increased by $0.74 \pm 0.18^{\circ} \mathrm{C}$ during the period $1906-$ 2005, while average $\mathrm{CO}_{2}$ levels increased from $280 \mathrm{ppm}$ before the industrial revolution to $379 \mathrm{ppm}$ in 2005 (IPCC, 2007). Moreover, it has been documented that this mean increase in temperature is produced by a rise in the minimum temperatures, resulting in a narrower diurnal temperature range (Easterling, 1997).

These changes in the abiotic parameters of the environment have a direct effect on biological systems. In the light of the current climatic variations the consequences have been analyzed across various levels of organization, seeking to establish causal relations between anthropogenic climate change and physical and biological impacts (Rosenzweig et al., 2008). At an ecosystem scale, the changes observed include for example: perturbations in seasonal events (such as migrations or time of reproduction) and shifts in biogeography and biodiversity (Rosenzweig et al., 2008). These effects can be traced to individual levels. Organisms live within a certain range of temperatures at which there is a coordinated functioning of molecular, cellular, and systemic processes (Pörtner and Farrell, 2008), and generally this is portrayed by a quadratic-shaped function. This function stands for the relation between these processes and temperature and it is represented by thermal performance curves. For each process there will be an optimal temperature, and two extreme points, i.e., an upper and a lower thermo-limit of performance. Particularly, ectothermic organisms are most vulnerable to thermal changes, given that their physiological processes are affected by temperature (Chown et al., 2010). Among ectotherms, terrestrial species are the ones most affected by environmental temperature changes due to the greater amplitude of change in their habitats compared to aquatic ecosystems. Terrestrial ectotherms comprise a large proportion of organisms and within this group there are some animals of great relevance to human health. Many of the worst epidemic diseases, such as Malaria, Chagas disease, Dengue, sleeping sickness, Leishmaniasis, Tick-borne encephalitis, among others, are transmitted by hematophagous arthropods that are ectothermic organisms. Thus, temperature will affect behavioral and physiological characteristics of diseases' vectors that are relevant to disease transmission.

Vector-borne diseases provide a fertile ground to address the general question of the causality between climate and biological change, and in order to understand fluctuations within this system (past, present, and future), information from different disciplines must be gathered. Having more accurate predictions from global change on the ecology of diseases, may enable more efficient health campaigns to be developed. Classical epidemiological parameters used to study disease transmission are based on the following factors: (i) survival and reproduction rates of vectors, (ii) intensity and temporal pattern of vector activity, and (iii) rates of development, survival, and reproduction of pathogens within vectors (Kovats et al., 2001). The vectorial capacity (VC) relates all of these factors as the number of infections resulting from the bites to one infected person per day through the following equation:

$$
\mathrm{VC}=\left(m a^{2} p^{\mathrm{n}}\right) /-\ln p
$$

where $m$, is the vector-host (human) ratio; $a$ is the biting frequency: the number of bites per vector per day; $p$ stands for the daily probability of survival; and $n$ for the development time of the parasite inside the vector. Another analogous epidemiological parameter widely used is the reproductive number $R_{0}$, which takes into account the same parameters as VC, plus the transmission coefficient from vertebrate to vector and from vector to vertebrate, as well as, the rate of host recovery from infection. Both expressions use information related to vector's biology. Variations on these parameters and their interactions will be reflected on the direction of the change of $\mathrm{VC}$ and $R_{0}$, i.e., a rise or decline in the disease (Rogers and Randolph, 2006). Vector survival rate may be affected in different directions, depending on the temperature and of how close of its thermal optimum the individual is. The vector-host ratio, on the other hand, depends both on vector's and human population density. The former depends upon parameters such as vector survival and reproduction rate, while the latter is driven mostly by socioeconomical factors, together with environmental characteristics. Consequently, it is not clear, weather to expect an increase or decrease in the vector-host ratio in response to increasing temperatures (Rohr et al., 2011). Finally, another important parameter affecting disease dynamics is the biting rate, which is inversely proportional to the amount of time between blood meals, 
and has a clear relationship with metabolic and nutrient conversion rates. Biting rate can be measured in the laboratory under controlled conditions, however, in the field only indirect estimations can be performed. For example, for triatomine bugs, vectors of Chagas disease, an indirect method is based on the distribution of bloodmeal weights from large vector samples, which would represent the distribution of times since last feeding (Rabinovich et al., 1979). Another method measures the presence of colorless urine that only occurs during the first few hours after feeding (Catalá, 1991). Nonetheless, these are indirect approaches and they have their limitations (see Rabinovich et al., 1979 and Catalá, 1991 for detailed explanation of the methods). Thus, it would be possible to directly measure the biting rate in the laboratory, without the limitations of an indirect measurement, and thereafter estimate the variation of biting frequency on the field based on the measured environmental temperature and the temperature sensitivity of the vector's metabolic rate (MR).

$Q_{10}$ is a measurement of temperature sensitivity, originally applied to rates of chemical reactions; it is the factor by which a rate changes in response to a variation of $10^{\circ} \mathrm{C}$, and it is a useful way to express the temperature dependence of a process. This factor may also be applied to whole organisms, for example, the sensitivity to temperature of MRs. Ectotherm's MRs are very sensitive to temperature, with $Q_{10}$ values around 2 , which means that a $10^{\circ} \mathrm{C}$ increase will double MR (Randall et al., 2001). Warmer temperatures speed up biochemical reactions, which in turn bring an increase in $\mathrm{MR}$ and nutrient transformation rate. As a consequence an increase on the frequency of biting would be expected. Thus, epidemiological mathematical models could use an estimated $Q_{10}$ of 2 or a calculated $Q_{10}$ based on measurements of MR at different temperatures from the species of interest as a simple way to account for changes in biting rate in response to thermal variations.

Thermal variations may have many effects on the physiology of vectors and consequently in vector-borne diseases transmission. Although common knowledge suggests that higher temperatures will lead to an increase in vector-borne diseases, recently this has been questioned and it has been proposed that a shift rather than an expansion in geographic range of disease, might occur. This consequence may be driven by variations in habitat suitability, which depends on climatic factors as temperature and humidity, as well as on barriers to dispersal and competition (Lafferty, 2009). Thermal changes are heterogeneous around the globe, as well as thermal tolerance among species, which is evidenced by their diversity across latitudes; at higher latitudes thermal tolerance is broader, while tropical organisms show more sensitivity to temperature changes (Chown and Nicolson, 2004). This implies that the consequences of an increase in environmental temperature will differ across latitudes (Deutsch et al., 2008) and species. Another factor that is generally left aside, are thermal daily variations. Most studies on the temperature effects on animal physiology are done under different constant temperatures, despite the fact that environmental temperature has a daily variation. However research has increasingly started to take more realistic daily variations into account. For example, in vectors of diseases, a variation on daily temperature preference has been observed in different species of kissing bugs, moreover, this preference is affected by the degree of starvation (e.g., Lazzari, 1991; Schilman and Lazzari, 2004). In addition, Paaijmans and collaborators showed that malaria transmission intensity is affected by the degree of daily temperature variations. Fluctuations around a low temperature increase rates of malaria parasite transmission, and mosquito development times and survival, while fluctuations around a high temperature slows the rates of these processes (Paaijmans et al., 2010). These results suggest that the molecular and physiological mechanisms underlying responses to temperature variations are complex and non-linear, and it is important to integrate them into current research of climate change impact.

In summary, several physiological factors affected by global warming should be considered to predict the dynamics of worldwide diseases' transmission. How these factors vary in response to thermal changes will be ultimately observed in modifications on the epidemiology of the disease. It has been observed that the underlying processes linking environmental temperature and rate of transmission diseases are not simple and direct linear relations. On one hand, the effects of daily temperature cycles on life history and survival of pathogens and vectors, as well as the differential sensitivity to thermal variations across different species and populations portray a complex system yet to be explored. On the other hand, and to construct the theoretical predictions of upcoming trends on vector-borne diseases simplified approaches might be useful. Thus, mathematical models could be developed taking into account the modulation of biting frequency by environmental temperature with the use of temperature sensitivity or coefficient $Q_{10}$ of the MR. In other words, based on the close relationship of ectothermic organisms between environmental temperatures, MRs, biting frequencies, and diseases' transmission rates, more specific models could be developed in future analyses.

Climate change is a global issue that should be approached in a global fashion. Therefore, we hope that the field of invertebrate physiology could provide more tools to improve the formulation of mathematical models to predict, among other things, upcoming trends on vector-borne diseases in a global climate change scenario.

\section{ACKNOWLEDGMENTS}

The authors thanks to Jorge Rabinovich, Claudio Lazzari, Gerardo de la Vega, and Maia Orsi for critical reading of an early version of the manuscript and ANPCyT/ Argentina (PICT2008-0268 and PICT20080035) for financial support. Carmen Rolandi has a Ph.D. fellowship and Pablo E. Schilman is researcher from CONICET/ Argentina.

\section{REFERENCES}

Arrhenius, S. (1896). On the influence of carbonic acid in the air upon the temperature of the ground. Philos. Mag. J. Sci. 41, 237-276.

Catalá, S. (1991). The biting rate of Triatoma infestans in Argentina. Med. Vet. Entomol. 5, 325-334.

Chown, S., Hoffmann, A., Kristensen, T., Angilletta, M., Stenseth, N., and Pertoldi, C. (2010). Adapting to climate change: a perspective from evolutionary physiology. Climate Res. 43, 3-15.

Chown, S. L., and Nicolson, S. W. (2004). Insect Physiological Ecology: Mechanisms and Patterns. New York: Oxford University Press.

Crowley, T. (2000). Causes of climate change over the past 1000 years. Science 289, 270-277.

Deutsch, C. A., Tewksbury, J. J., Huey, R. B., Sheldon, K. S., Ghalambor, C. K., Haak, D. C., and Martin, P. R. (2008). Impacts of climate warming on terrestrial ectotherms across latitude. Proc. Natl. Acad. Sci. U.S.A. 105, 6668-6672. 
Easterling, D. R. (1997). Maximum and minimum temperature trends for the globe. Science 277, 364-367.

IPCC. (2007). Climate Change 2007: The Physical Science Basis. Working Group I Contribution to the Fourth Assessment Report of the IPCC. Cambridge: Cambridge University Press.

Kovats, R.S., Campbell-Lendrum, D. H., McMichel, A. J., Woodward, A., and Cox, J. S. H. (2001). Early effects of climate change: do they include changes in vectorborne disease? Philos. Trans. R. Soc. Lond. B Biol. Sci. 356, 1057-1068.

Lafferty, K. D. (2009). The ecology of climate change and infectious diseases. Ecology 90, 888-900.

Lazzari, C. R. (1991). Temperature preference in Triatoma infestans (Hemiptera: Reduviidae). Bull. Entomol. Res. 81, 273-276.

Paaijmans, K. P., Blanford, S., Bell, A. S., Blanford, J. I., Read, A. F., and Thomas, M. B. (2010). Influence of climate on malaria transmission depends on daily temperature variation. Proc. Natl. Acad. Sci. U.S.A. 107, 15135-15139.
Pörtner, H. O., and Farrell, A. P. (2008). Physiology and climate change. Science 322, 690-692.

Rabinovich, J. E., Leal, J. A., and Feliciangeli de Piñero, D. (1979). Domiciliary biting frequency and blood ingestion of the Chagas's disease vector Rhodnius prolixus Ståhl (Hemiptera: Reduviidae), in Venezuela. Trans. R. Soc. Trop. Med. Hyg. 73, 272-283.

Randall, D., Burggren, W., and French, K. (2001). Eckert Animal Physiology: Mechanisms and Adaptations. New York: W. H. Freeman.

Rogers, D. J., and Randolph, S. E. (2006). Climate change and vector-borne diseases. Adv. Parasitol.62, 345-381.

Rohr, J. R., Dobson, A. P., Johnson, P. T. J., Kilpatrick, A. M., Paull, S. H., Raffel, T. R., Ruiz-Moreno, D., and Thomas, M. B. (2011). Frontiers in climate change-disease research. Trends Ecol. Evol. (Amst.) 26, 270-277.

Rosenzweig, C., Karoly, D., Vicarelli, M., Neofotis, P., Wu, Q., Casassa, G., Menzel, A., Root, T. L., Estrella, N., Seguin, B., Tryjanowski, P., Liu, C., Rawlins, S., and Imeson, A. (2008). Attributing physical and biologi- cal impacts to anthropogenic climate change. Nature 453, 353-357.

Schilman, P., and Lazzari, C. R. (2004). Temperature preference in Rhodnius prolixus, effects and possible consequences. Acta Trop. 90, 115-122.

Received: 14 February 2012; accepted: 14 March 2012; published online: 29 March 2012.

Citation: Rolandi C and Schilman PE (2012) Linking global warming, metabolic rate of hematophagous vectors, and the transmission of infectious diseases. Front. Physio. 3:75. doi: 10.3389/fphys.2012.00075

This article was submitted to Frontiers in Systems Biology, a specialty of Frontiers in Physiology.

Copyright $(2012$ Rolandi and Schilman. This is an openaccess article distributed under the terms of the Creative Commons Attribution Non Commercial License, which permits non-commercial use, distribution, and reproduction in other forums, provided the original authors and source are credited. 